STUDIA HUMANITATIS JOURNAL, 202I, I (I), pp. I-I9

ISSN: 2792-3967

D0l: https://doi.org//0.5370l/shj.vlil.10

\title{
LA GESTIÓN DEL TIEMPO DURANTE EL CONFINAMIENTO: EL CASO DEL ALUMNADO UNIVERSITARIO $0^{1}$
}

\author{
Francisco Javier Aroca Cifuentes \\ Universidad de Castilla-La Mancha, España \\ ORCID: 0000-000I-5684-2678 \\ franciscoj.aroca@uclm.es
}

(1) (8)

\begin{abstract}
Resumen | El presente artículo ofrece una panorámica sobre cómo ha gestionado su tiempo vital una muestra de estudiantes de la Universidad de Castilla-La Mancha -España- durante el período del estado de alarma. El tránsito de unas vivencias auspiciadas por la normalidad a un escenario sin precedentes en nuestras vidas, marcado por la pandemia de la COVID19 supone un cambio de modelos comportamentales generales y muy especialmente de forma particular en el lapso temporal del confinamiento domiciliario. Así, este análisis exploratorio sirve para ilustrar cómo el perfil de estudiantado universitario ha organizado sus vidas, compatibilizando sus deberes como estudiantes con la realización de otras actividades, lo que permite conocer también cómo son sus pautas de socialización y sus tiempos de ocio y de descanso. Se plantea, pues, una investigación cualitativa cuyo objetivo principal es conocer en un sentido amplio cómo vivencia este perfil estudiantil la nueva realidad impuesta durante el estado de alarma, bajo la obligatoriedad de permanecer en el hogar. El análisis, sustentado en la entrevista en profundidad, recoge evidencias de los efectos que tiene el confinamiento en la capacidad organizativa del alumnado. Se demuestra que dicha capacidad organizativa está en función de factores personales, ambientales y de la continuidad de la docencia online. Por otra parte, cabe destacar cómo la situación de confinamiento llega a afectar negativamente a la capacidad de concentración y a la percepción de productividad, siendo necesario realizar una mayor inversión de tiempo en el aprendizaje.
\end{abstract}

Palabras clave: Gestión del tiempo, Confinamiento, Educación, Juventud.

Abstract | This article offers an overview of how a sample of students from the University of Castilla-La Mancha Spain- has managed their vital time during the period of the state of alarm. The transition from experiences sponsored by normality to an unprecedented scenario in our lives, marked by the COVID-19 pandemic, supposes a change in general behavioral models and especially particularly in the time period of lockdown. Thus, this exploratory analysis serves to illustrate how the profile of university students has organized their lives, reconciling their duties as students with carrying out other activities, which also allows us to know what their socialization patterns and their leisure and rest times. Therefore, a qualitative research is proposed whose main objective is to know in a broad sense how this student profile experiences the new reality imposed during the state of alarm, under the obligation to stay at home. The analysis, supported by the in-depth interview, collects evidence of the effects that confinement has on the organizational capacity of the students. It is shown that said organizational capacity is a function of personal and environmental factors and the continuity of online teaching. On the other hand, it should be noted how the confinement situation negatively affects the ability to concentrate and the perception of productivity, requiring a greater investment of time in learning.

' Recibido/Received: 03/05/2021

Aceptado/Accepted: 07/06/2021 


\section{| Introducción |}

La propagación de la COVID-19 a todos los rincones del planeta durante el año 2020 ha esbozado un nuevo escenario al que hemos tenido que adaptarnos con diferentes estrategias e incluso improvisaciones en todos los ámbitos de nuestras vidas. Este es un difícil período marcado por una pandemia que se dilata en el tiempo desde hace más de un año y que tiene profundas implicaciones: económicas, políticas, sociales, sanitarias, etc. A su vez, esta nueva realidad también ha contribuido a acelerar algunos procesos de cambio que ya se venían gestando con anterioridad. Se trata de unas dinámicas evolutivas que propician transformaciones en diferentes áreas de nuestras vidas: laboral, educativa, social, personal, etc. Como ejemplo clave de este progreso, destaca el imperante protagonismo de las nuevas tecnologías, que se focaliza en nuestro día a día mediante un mayor auge en su utilización y se evidencia en una tendencia creciente en actividades como el teletrabajo, así como en una conectividad de usuarios superior.

Ante unos cambios de tal magnitud, acrecentados en parte por la propia evolución de la pandemia, es de rigor poner en valor el papel que deben desempeñar las Ciencias Sociales en este momento de cambio. Ya que deben tomar parte activa en el análisis de cómo están afectando estas dinámicas a la sociedad en su conjunto, así como determinar las repercusiones específicas que pueden manifestarse en algunos colectivos en concreto. No obstante, ante esta compleja situación sanitaria, numerosas revistas científicas han publicado trabajos puntuales o volúmenes monográficos con la finalidad de reflexionar sobre lo acontecido, e incluso para detectar claves en futuros escenarios marcados por otras posibles pandemias que estén por venir (Cabero y Valencia, 2021). Ya que, por su significación, las pandemias no son única y exclusivamente fenómenos biológicos, sino que también lo son sociales, en la medida en que existen implicaciones diversas en la sociedad y se muestran diferentes grados de incidencia en las estructuras sociales. (Fernández, 2020).

Tras este esbozo inicial en lo relativo a la crisis sanitaria y a sus implicaciones generales, vamos a centrar la atención analítica en el ámbito educativo. Siendo el curso académico 2019/2020 un año en el que la educación presencial, ha realizado un esfuerzo titánico y sin precedentes por parte de todos los agentes implicados para continuar con el proceso formativo -docentes, alumnado y por supuesto familias en el acompañamiento de los más jóvenes-. Aunque, por otra parte, el confinamiento también ha evidenciado las múltiples brechas persistentes en el propio sistema educativo, algo que queda reflejado en las distintas desventajas de origen que asumen algunos colectivos sociales (Tarabini, 2020). De ahí que, tanto en un primer momento como posteriormente, ante esta situación, se cuestiona la enseñanza semipresencial o no presencial en los centros formativos que son eminentemente presenciales (García-Planas y Taberna, 2021).

Hechas estas aclaraciones previas, cabe destacar que este trabajo centra su atención en un colectivo muy concreto: la población universitaria que estudia en modalidad 
presencial. Siendo el objeto de estudio la gestión del tiempo que realiza este alumnado durante el confinamiento. Ya que es fundamental profundizar en la importancia de la gestión del tiempo para alcanzar con éxito los objetivos académicos propuestos (Garzón y Gil, 2018). Siendo precisamente en estos escenarios ajenos al aula donde más peso tiene la autonomía personal y la necesaria capacidad de organizar el tiempo de una forma eficiente (Feito, 2020).

Tradicionalmente, el tiempo siempre es considerado como un valor escaso y por lo tanto muy apreciado, siendo ensalzado este pensamiento en numerosas expresiones populares. Pero ante una situación como el confinamiento, la percepción -elemento subjetivo- del tiempo cronológico -hecho objetivo- parece ser distinta respecto a tiempos previos, debido al condicionamiento espacial. Siendo de suma importancia la experiencia interior del transcurso del tiempo para entender el comportamiento humano (Bayés, 2007). No obstante, ante esta propuesta de investigación, es preciso tener en consideración el marco contextual donde se desarrolla la misma, el escenario privilegiado de unas sociedades tecnológicamente avanzadas, también denominadas como sociedades de la información, que no necesariamente implican una sociedad del conocimiento (Área y Pessoa, 2012). No obstante, a partir de ese análisis concreto de la gestión del tiempo, se vislumbran otros aspectos interrelacionados de sumo interés, y que sirven para comprender mejor una situación tan excepcional. En cuanto a las personas participantes, se trata de un perfil eminentemente joven, aunque muy maduro en muchos aspectos. Así como garante de un elevado nivel cultural y que ostenta un avanzado conocimiento tecnológico, factores que le confieren por una parte, una notable capacidad crítica, así como una nada desdeñable habilidad competencial en materia tecnológica.

Para finalizar este apartado introductorio, es preciso destacar que algunos de los resultados de este artículo han sido presentados al II Encuentro Intercongresual de Sociología del Tiempo, organizado por el Comité de Investigación 27 -Sociología del Tiempo- de la Federación Española de Sociología.

\section{Hipótesis}

Una vez esbozado someramente el marco contextual en el que se ubica este trabajo, así como la temática y los sujetos implicados en el análisis, se procede a especificar las dos hipótesis de investigación en base a las siguientes preguntas de investigación: ¿Condiciona de forma negativa el confinamiento en las formas de organización temporales del alumnado? ¿Influye dicho confinamiento en una percepción personal de menor productividad? Ante estas preguntas se plantean las siguientes hipótesis:

- La primera hipótesis sostiene que, el confinamiento estricto condiciona negativamente en las formas de organización temporal del alumnado. 
- La segunda hipótesis afirma que, el período de confinamiento incide en una percepción subjetiva de menor productividad.

La selección de estas hipótesis conecta el factor estudio, que es la actividad central de este perfil de alumnado universitario, con el condicionamiento espacial así como con la capacidad de gestionar su tiempo. De modo que, según la lógica propuesta en ambas hipótesis, el confinamiento incide de una forma negativa en el alumnado. Por una parte, se sostiene que influye de forma perniciosa en su organización del tiempo y por otra parte, que también va a influir de forma negativa en su percepción de un menor desarrollo productivo.

\section{| Objetivos |}

Para dar respuesta a las hipótesis de investigación planteadas, se establece un objetivo principal: Conocer en un sentido amplio cómo vivencia este perfil estudiantil la nueva realidad impuesta durante el confinamiento, la obligatoriedad de permanecer en el hogar. A partir de este objetivo principal, se articulan los siguientes objetivos específicos: Conocer cómo está influyendo el confinamiento en su gestión del tiempo y cómo está afectando al conjunto de su proceso educativo; Indagar en el grado de utilización de las nuevas tecnologías en esta fase y Averiguar cómo son sus procesos de socialización. Queda patente la utilidad de este análisis en la medida en que sus resultados son también extrapolables a otros alumnados de perfiles socioeconómicos similares.

\section{| Estrategia metodológica |}

Para dar respuesta a los objetivos señalados anteriormente, se plantea un estudio cualitativo con alumnado de dos grados de la Universidad de Castilla-La Mancha: Educación Social -segundo curso-, del campus de Cuenca y Criminología -primer curso, del campus de Albacete. La herramienta escogida para alcanzar los objetivos propuestos es la entrevista en profundidad, debido a las notorias virtudes que ofrece este método: plasticidad a la par que profundidad. Tras realizar un llamamiento en ambos grados a participar en esta investigación, se ha entrevistado a diecisiete estudiantes que voluntariamente han accedido a narrar sus experiencias vitales en base a las preguntas establecidas en un guion flexible previamente diseñado. Las entrevistas han sido realizadas a través de la plataforma de comunicación Microsoft Teams, vía Internet. Se ha grabado exclusivamente el audio de las mismas y posteriormente han sido transcritas y analizadas por temáticas. Entre los aspectos tratados en estas narraciones destacan aspectos clave, como son: la valoración de las clases virtuales en comparación con las presenciales, el estudio limitado al hogar y aparejado en ocasiones a unas condiciones dificultosas, la mayor complejidad en la coordinación de trabajos académicos, los mecanismos de socialización que se despliegan ante las limitaciones impuestas, el grado de importancia que ostentan sus aficiones $y$ otras formas de recreo durante el 
confinamiento, así como la influencia del encierro en el desarrollo de actividades puramente funcionales: alimentación y descanso.

Según Valles (2000), se debe entender el diseño de una investigación cualitativa como un proceso por el cual se da forma a una investigación mediante fases específicas, lo que implica también una toma de decisiones, tanto teóricas como metodológicas que el investigador realiza en tres etapas de la investigación -previamente, durante y después del estudio- (Díaz, 2018). De ahí el nivel de adaptación llevado a cabo durante todo el proceso de investigación.

De cara a establecer una clasificación geográfica de perfiles entrevistados, se establece como municipio de residencia la localidad donde vive cada sujeto durante el confinamiento. Así, se establece la diferenciación entre: zonas rurales -municipios de menos de 10.000 habitantes- y zonas urbanas -superior o igual a 10.000 habitantes-, siendo nueve participantes los residentes en zonas urbanas y ocho en municipios rurales. Prevalecen las residencias en Castilla-La Mancha, aunque también hay municipios de otras regiones.

La tabla 1 (ver anexos) ofrece información específica de la muestra: edad y género de la persona entrevistada. Así como datos relevantes de los hogares en que residen durante el confinamiento: número de convivientes y su grado de relación, número de personas en activo, percepción de prestaciones, metros cuadrados de la vivienda, existencia de patio, etc. Cabe señalar que todas las personas entrevistadas son de nacionalidad española, pertenecientes a lo que se conoce como clase media. No obstante, la pandemia ha incidido directamente en muchos de estos hogares familiares; siendo varios los que tienen a alguno de sus miembros afectados por un ERTE (Expediente Regulador de Empleo Temporal), de ahí el bajo nivel de trabajadores/as en activo reflejado.

\section{| Resultados |}

En este apartado se describen en primer lugar las estrategias organizativas de los hogares, así como el nivel y frecuencia con la que se informan. En segundo lugar, se profundiza en el significado del tiempo. En tercer lugar, se expone la manera en que se gestiona el tiempo, vinculando este factor con el rendimiento académico. En cuarto lugar, se particularizan otros aspectos como son el tiempo de descanso y los cambios experimentados en la forma de organizar sus rutinas.

Antes de proceder a detallar estos aspectos del análisis, es preciso conocer previamente, aunque sea a grandes rasgos, los contextos en los que desarrollan sus vidas las personas entrevistadas. Una parte importante de este alumnado estudia fuera de su municipio habitual de residencia. Así que, algunos/as regresan al hogar de sus progenitores con anterioridad a la declaración del estado de alarma, mientras que otros/as no pueden retornar por la entrada en vigor de dicho decreto. No obstante, en ambos casos se 
precisa de una adaptación organizativa tanto individual como colectiva en el hogar ante una situación que imposibilita salir al exterior con normalidad.

Frecuentemente se afronta el encierro como un reto grupal a superar por parte de los miembros de la vivienda y para ello, en algunos casos se establece un reajuste en el reparto de responsabilidades domésticas. Las tareas del hogar son un elemento de análisis muy interesante y que habitualmente sirve para diferenciar los usos del tiempo en el hogar según el género, evidenciando la mayor carga de trabajo que desempeñan las mujeres en el ámbito privado, tal y como se reflejan numerosos estudios.

En esta muestra se percibe una continuidad de responsabilidades asociadas a perfiles que ya estaban asignadas previamente. Por lo tanto, de forma generalizada no se aprecian grandes cambios. En los hogares familiares no se percibe una gran diferencia en el reparto de tareas durante el confinamiento, siendo las mujeres las que más tareas realizan. Como excepción, destacan los casos concretos en los que uno o varios miembros deben trabajar fuera del hogar y los que no lo hacen apoyan más de lo habitual en determinados quehaceres. Sin embargo, resulta llamativo el hecho de que la realización de la compra -actividad que implica cierto grado de peligrosidad por salir del entorno seguro que es el hogar- está asignada únicamente a una persona del hogar, por lo general el padre. Mientras que en los hogares compuestos por compañeros de piso, las tareas se distribuyen de forma equitativa, igual que con anterioridad y se alterna la actividad de realizar la compra.

No, problema no. Mi madre que se queja de que hace todo, aunque le ayudamos.

De hecho, es eso. Como mi padre está más tiempo en casa, si tiene que hacer la comida o la cena o poner una lavadora lo hace, cosa que antes no lo hacía. (R3)

Un aspecto fundamental es el gran peso que tienen las noticias durante el confinamiento. Debido a lo alarmante de la situación inicial tras la declaración de la pandemia, el volumen de tiempo que dedica la población a informarse es muy superior al habitual, al menos en un primer momento. El seguimiento de las noticias, tanto en medios oficiales -noticias en televisión o prensa digital- como en redes sociales es muy notorio, llegando a saturar el exceso de información. Además, preocupa la coexistencia de noticias reales con falsas -fake news-, relativamente frecuentes en algunas redes sociales. De ahí la necesidad de discernir contenidos verídicos de otros no contrastados para informarse. Mientras que en algunos casos se opta por reducir la exposición a la información por una cuestión de bienestar psicológico.

Al principio, el primer fin de semana, que fue una locura, estuve bastante conectado por ver un poco que pasaba. Sobre todo, como fue cuando saltó el estado de alarma, y luego yo ya me aislé un poco (...) Luego, también porque en los grupos de WhatsApp, les dije que era demasiada información y sobre todo sin ningún filtro. (...) He intentado informarme de lo que nos afecta (...) De bulos he pasado bastante porque sabía que no iba a ser información real. (U1)

Tras este aproximamiento inicial, es el momento de centrar la atención en el elemento analítico clave de este trabajo: el tiempo. Cabe señalar en un primer momento, que no 
siempre percibimos el tiempo del mismo modo en todas las etapas de nuestra vida, ya que puede depender de nuestra edad o de otros factores internos o externos. Esta percepción del tiempo también difiere en algunos períodos concretos; como cuando estamos de vacaciones, por no tener que atender a unas rutinas marcadas por las obligaciones imperantes. De ahí que el tiempo parece fluir también de un modo distinto durante el confinamiento; en la medida en que no existe la posibilidad de realizar actividades fuera del hogar, ni se establece la obligación de acudir al aula o a otras citas. Por lo tanto, prevalece una extraña percepción de quietud del tiempo, al permanecer constantemente en un mismo espacio físico durante un largo período. A esta apreciación se suma la frustración por las sucesivas prorrogas del estado de alarma, motivo que genera más desasosiego en ese fluir temporal y que tiene un efecto claramente negativo en el estado anímico de prácticamente todas las personas.

Al principio era un poco agobiante, venga dos semanas. Pero cada vez que nos decían otras dos y otras dos, como que te agobiabas un poco más. Ha habido semanas peores y otras mejores. No vas a estar pensando todo el día estoy encerrada. Intentaba tener la cabeza ocupada con trabajos, escuchando música, trabajas un poco la paciencia. Bueno, si tengo que estar otras dos semanas, vamos a estar. (U4)

Según Amando de Miguel e Iñaki de Miguel, "resulta difícil desarrollar las faenas de la vida cotidiana sin saber la hora y la fecha en la que se está" (De Miguel y De Miguel, 2014). Bajo este planteamiento, se evidencia la necesidad humana de ubicarnos en el tiempo cronológico por medio de algunos referentes claros para poder organizar nuestras actividades de forma exitosa. Sin atender a horarios ni fechas, difícilmente se pueden plantear unos objetivos precisos debido a esa falta de concreción con la misión a cumplir. Esto sucede independientemente de que el compromiso lo marque el propio individuo o venga impuesto por otras personas o instancias. No obstante, a pesar de contar con calendarios y relojes, dicha percepción del tiempo es distinta durante el confinamiento, tal y como se apuntaba anteriormente. Así que, no es de extrañar que se busquen algunas estrategias propias para distinguir los días laborables de la semana de los de descanso, aportando así cierta coherencia diferencial. Mismamente, se percibe en todos los casos que entre semana se dedica más tiempo al estudio y seguimiento de las sesiones lectivas. Mientras que de forma generalizada, el fin de semana se dedica mayoritariamente al descanso, aunque también lo aprovechen para estudiar algunas personas.

Al principio era muy dispersa, no me marcaba ninguna rutina. No se sabía cómo
iban a pasar las cosas, ni si íbamos a volver. Eso unido a que el tiempo era muy
abstracto... Parece que no pasa, pero pasa más rápido. Al principio intentaba
hacer cosas diferentes para que se notara que era sábado, me echaba algún
producto en el pelo o me pintaba las uñas, para que no fuera lo mismo que de
lunes a viernes. (U4) 
No obstante, en un primer momento, ni si quiera existe un compromiso horario que atender debido a la suspensión temporal de la docencia. Siendo esta primera fase un periodo general de bastante incertidumbre, en el que tampoco se conoce cómo ni cuándo se dará continuidad a la docencia.

Las pautas de sueño se han visto alteradas durante el confinamiento en gran parte de este colectivo. La falta de una actividad concreta en un momento inicial que implique el establecimiento de una rutina supone en un primer momento cierto relajamiento generalizado. No obstante, en cuanto al sueño, es evidente que hay situaciones de todo tipo. Desde perfiles que con anterioridad al confinamiento dormían muy pocas horas, a otros que tienen unas pautas de sueño consideradas como normalizadas. Sin embargo, a prácticamente la totalidad de la muestra el confinamiento les ha trastocado el sueño de un modo u otro.

Siento como que no duermo excesivamente bien, porque sabes que al día siguiente te toca lo mismo y así día, tras día, tras día. (U9)

Además, es bastante generalizado el hecho de trasnochar, especialmente cuando comparten piso compañeros o amigos. Aunque esta práctica, no se circunscribe únicamente a ese caso, ya que también está extendida a los hogares en los que se convive con la familia. Por lo tanto, se evidencia un desorden importante en lo relativo al sueño. Se podría teorizar sobre la incidencia de cómo afectan diferentes factores: la falta de actividad en el exterior, la ausencia de ejercicio físico y el uso excesivo de dispositivos electrónicos a cualquier hora. Siendo estos factores señalados por ellos mismos como perniciosos para el correcto descanso nocturno.

Al principio, la mala rutina de acostarnos a las 4.00 o las 5.00. (...) El cambio fue cuando empezasteis a dar clase, porque los primeros días no había clase. Es que hay una que la tenemos a las nueve de la mañana. ¿Para qué me voy a dormir, si me tengo que levantar en dos horas? Al empezar las clases, empezó un horario habitual. (R8)

Ante la primera hipótesis, el confinamiento condiciona negativamente la organización del estudiantado; se aprecia que tienen un gran peso los rasgos de la propia personalidad. Así, las personas que afirman ser más organizadas han evidenciado una adaptación más rápida y menos traumática desde un primer momento.

Soy una persona bastante organizada y lo he llevado bastante bien. (...) He estado bastante solo en casa, pero comunicándome con muchas personas a través de la red. Creo que lo estoy llevando bastante bien. Me he organizado los días (...) Tardes a las clases y a tocar (la guitarra) y a leer. (U1)

Mientras que, por otra parte, las personas que se consideran menos organizadas, se han permitido un período de inactividad inicial o han priorizado la realización de otras actividades o descansar en mayor medida. Este cambio de escenario marcado por el confinamiento supone una dificultad para este último grupo, en la medida en que le exige una mayor autodisciplina. 
No soy una persona muy organizada. Esto me ha fastidiado en el sentido de que si vas a la universidad tienes tu horario. Si. Ahora que son online las clases, como que lo he ido un poco reestructurando. (U7)

No obstante, el confinamiento supone de forma generalizada una importante alteración de la organización temporal $y$, por lo tanto, les dificulta enormemente centrar su actividad. Al no evidenciarse urgencias en un primer momento, se tiende a la procrastinación, ya que es factible retrasar lo evitable.

Al principio no me quise organizar nada porque voy muy por libre. Si me apetece hacer algo lo hago y si no, no. (U5)

De este modo, se establece un momento clave que supone un punto de inflexión en la forma de organizar el tiempo al retomar la docencia. Tras los desajustes horarios anteriormente señalados de buena parte del alumnado, se produce una vuelta a los hábitos diurnos. Aunque en algunos casos, también se lleva a cabo el cambio motivado por los consejos de algún miembro del hogar. Además, de suponer un aliciente al hábito organizativo del alumnado, las clases también han significado un apoyo, al establecer una comunicación fluida con el profesorado. En añadidura, la comunicación es un factor clave para evitar la sensación de aislamiento que suele producirse en los modelos de enseñanza a distancia; efecto agudizado, además, por el notorio impacto psicológico que la reclusión forzosa por la pandemia puede provocar. (Pérez-López et. al., 2021).

Me lo dijo mi madre. Esto que llevas no es vida. Un día me dormí y estuve todo el día dormida, pero ya para coger la rutina de dormirme temprano y ahora sí que la tengo. Fue hace tres semanas como mucho, cuando ya he empezado con el tema del estudio básicamente. Después de la Semana Santa, después. (R6)

Para retomar el ritmo diario perdido, en algunas ocasiones se opta por adoptar soluciones drásticas como es el caso de dormir durante todo un día o bien la opción contraria, permanecer en vela durante toda una noche. Prácticas que cuando menos resultan bastante curiosas como procesos adaptativos.

Al principio lo llevaba normal. Yo me encierro en mi habitación y no me importa. Pero hubo una temporada que perdí el sueño. Me compré un puzle y me podía tirar hasta las 5.00 con el puzle y luego me levantaba a la hora de comer o más tarde. Hubo una semana, la de Semana Santa que perdí total el horario. Y me levantaba a las cuatro de la tarde. Pero un día me tiré toda la noche despierta y ya normal. (R3)

Una vez matizado el aspecto temporal, es el momento de centrar la atención en la actividad esencial que lleva a cabo el alumnado: sus estudios. No obstante, esta categoría está sujeta a los vaivenes temporales anteriormente señalados. Además, en el hogar, las distracciones son abundantes, tanto por las múltiples opciones que se posibilitan en forma de entretenimiento, como por las posibles interrupciones que pueden producirse en unos hogares en los que todos o casi todos sus miembros están compaginando diferentes actividades laborales o estudiantiles. De ahí que a muchos sujetos les cueste 
adaptarse para ser todo lo productivos que desearían. Ante este nuevo modelo de aprendizaje, es preciso disponer de unos recursos mínimos -herramientas tecnológicas, conexión a Internet con suficiente velocidad y también estabilidad-, así como competencias de autogestión en el proceso de aprendizaje a distancia, que engloban entre otras desde la competencia lecto-escritora hasta una adecuada gestión del tiempo (Cabero y Valencia, 2021).

En algunas ocasiones, ante la imposibilidad de poder alcanzar un grado óptimo de concentración durante el día, se opta por estudiar en horarios de vigilia, cuando reina el silencio tanto en el propio hogar como en el vecindario.

Una semana estudiaba por las tardes cuando no tenía clases, otras de madrugada. Es que como tengo el sueño descontrolado, pues cuando estoy despierta. (...) Tienes que aprovechar que no haya ruido en casa o en la de los vecinos. No depende solo de tu propia organización, depende de lo de alrededor. La verdad es que suelo estudiar más bien por la noche, porque hay silencio y duermen todos. (U6)

Es oportuno indagar en cómo considera el alumnado que le va a afectar el nuevo modelo de docencia y evaluación a sus expectativas de resultados académicos. De ahí que se incida especialmente en esta cuestión para tener una noción de lo que esperan que suceda. Así pues, muchas personas afirman que el confinamiento va a incidir de forma negativa en sus notas. Esto lo achacan a varios motivos. En algunos casos, las personas que habitualmente estudian en bibliotecas consideran que rinden menos en el hogar. Otras personas también señalan que al no poder salir de casa les cuesta más desconectar del estudio porque no cambian de entorno, aunque lleven a cabo actividades de ocio.

Estoy muy acostumbrada a estudiar en la biblioteca. De por la mañana estudiar en la biblioteca y al estar en casa... Mi casa la atengo asociada a relajarme, olvidarme un poco de la universidad. Y ahora, al estar todo el tiempo aquí... es el mismo lugar para relajarme que para estudiar. Yo creo que me va a costar más. Más horas. Me cuesta más concentrarme. Si allí tardaba media hora en coger el ritmo, aquí tardo una hora. Ha cambiado bastante. (R5)

La gran mayoría del alumnado considera que dedica una mayor cantidad de tiempo al estudio, así como a la realización de trabajos durante el confinamiento. Aunque perciben que no siempre se aprovecha ese tiempo invertido, debido entre otras cuestiones a las múltiples distracciones presentes en el hogar, que les dificultan concentrarse lo suficiente en su tarea en muchas ocasiones.

A mí me pasa que leo algo y digo es que no recuerdo lo que he leído. Yo creo que sí me va a repercutir bastante.

Pregunta: ¿Dedicas más horas al estudio?

Sí, desde luego que ahora tengo que echar más horas. Ahora empleo más horas, porque si no, no lo logro entender. (R2) 
Sin embargo, algunas personas afirman que dedican incluso menos tiempo al estudio en la situación actual. Otra cuestión muy relevante es la sensación de pérdida de efectividad de la docencia en remoto. A pesar de ser las clases un factor de gran importancia tal y como se ha demostrado, no satisfacen plenamente las expectativas del alumnado. De ahí que sea muy significativa la dificultad de seguir la docencia en línea a pesar de contar con los medios tecnológicos (Diez-Gutierrez et al., 2020).

¿Horas?... Yo creo que menos, antes ibas a la biblioteca y de ver a la gente estudiando te obligabas. Ahora en tu escritorio y voy a mirar el móvil, te concentras más porque estás sola, pero es como que también es diferente porque no te obligas, bueno tengo más tiempo para estudiar. Clases: Sí y no. Es muy distinto estar frente a un ordenador escuchando, sin el cara a cara. Porque estamos acostumbrados a eso y gracias a eso, estás más pendiente. (R7)

Tras un análisis de la relación entre tiempo y estudio, es el momento de centrar la atención en los aspectos lúdicos. Una de las señas de identidad de las sociedades plenamente desarrolladas es la elevada cantidad de tiempo que la población dedica al ocio y al consumo. Si bien en períodos anteriores la mayor parte del tiempo de todos los individuos se orientaba a la producción, en el presente el ámbito del consumo ha alcanzado cotas sin precedentes de dedicación de recursos, tanto económicos como temporales. No se trata de un consumo exclusivo de productos, sino también del consumo de ocio, que es fundamental en estas sociedades tecnológicamente avanzadas.

La gran cantidad de avances que posibilitan las nuevas tecnologías, a través de Internet, permiten múltiples y diversas opciones de esparcimiento. En un sentido amplio, durante el confinamiento, se ha posibilitado el realizar visitas virtuales a museos, seguir conciertos, visualizar obras de teatro en streaming, etc. De forma que personas de todas las edades y con diferentes preferencias en cuanto a contenidos, han encontrado entretenimiento adaptado a sus gustos en los dispositivos electrónicos. Con relación a esta diversidad de opciones de ocio a nuestro alcance y a las múltiples posibilidades de comunicación gracias al fácil y bastante generalizado acceso a Internet, cabe plantearse lo diferente que hubiese sido la vivencia del confinamiento en esta pandemia provocada por la COVID-19, de haberse producido dos décadas antes; en un período en el que no había un nivel de desarrollo tecnológico tan avanzado ni popularizado y en el que los sistemas de comunicaciones también eran mucho más básicos. Las nuevas tecnologías han posibilitado la continuidad de buena parte de la actividad laboral y educativa, a pesar de las dificultades. Sin embargo, este escenario no es totalmente generalizado en todas las capas de la sociedad. Es cierto que la vida moderna en la que estamos inmersos ha permitido suavizar en gran medida esta experiencia, en el caso de las personas que cuentan con los medios y los conocimientos que posibilitan el uso de dichos dispositivos electrónicos. Mientras que se evidencia una brecha digital en los individuos de más edad -también más vulnerables a nivel sanitario-, o en el caso de los perfiles con menos recursos económicos, que también carecen de esos recursos tecnológicos. 
Cabe destacar también, que el tiempo que se dedica a las amistades es muy notorio durante el confinamiento. Se emplea un lapso de tiempo, generalmente al final de la tarde para poder charlar o verse a través de una pantalla. Estas relaciones efectuadas por estos medios son consideradas como mucho más frecuentes que en condiciones de normalidad. Ante la carencia del contacto físico, tanto con familiares como con amistades o pareja, se aprecia un notorio auge en el uso de diferentes herramientas o aplicaciones que posibilitan la realización de videollamadas o reuniones grupales de forma virtual. Estos medios posibilitan la difusión tanto de la imagen en movimiento como del audio en tiempo real, siendo una opción de comunicación muy atractiva. Además, estos recursos posibilitan el hecho de retomar actividades, como por ejemplo quedar con alguna persona o en grupo para tomar algo, obviamente de forma virtual. Se establece de este modo, un espacio de encuentro que palia el distanciamiento social marcado por la pandemia y propicia canales de comunicación fluidos. El empleo de algunos de estos recursos era más bien anecdótico y poco extendido anteriormente, pero aquí se evidencia la importancia de la imagen en la sociedad actual, el aspecto visual ligado al momento presente.

Porque quieras que no, es ese momento del día en el que te despejas para hablar con tus amigos, con tu gente. $Y$ dentro de lo que cabe, los notas más cerca. No es lo mismo hablar por WhatsApp -mensajes de texto- que por una videollamada. (U4)

También se extrapola esta relación de mayor cercanía en el caso de no convivencia con alguno o los dos progenitores; e incluso con otros miembros de la familia con los que se puede llevar a cabo una comunicación más directa a través de videollamadas.

Yo creo que he hecho más videollamadas ahora que en toda mi vida. (...) De hecho, muchas más de lo normal. Llamo a mi madre y a mi padre más. Todas por Zoom (videollamadas), que ahora está súper de moda. (U7)

Una de las peculiaridades del confinamiento en España ha sido el salir a aplaudir desde ventanas y balcones a las 20.00 horas. Esta práctica colectiva es una muestra de reconocimiento a determinados perfiles profesionales, principalmente sanitarios, aunque también extensible a cuerpos y fuerzas de seguridad del Estado, así como a personal esencial-limpieza, supermercados, etc.- Sin embargo, este acto simbólico va más allá de esa acción que denota gratitud a esas personas, puesto que ha tenido otras repercusiones no buscadas. Al tratarse de un acto sincronizado y coincidir habitualmente con las mismas personas del vecindario, se establece la tendencia de saludar o charlar un rato, e incluso llevar a cabo otro tipo de acciones colectivas más complejas en algunas ocasiones -juegos, coreografías, etc.-. Se trata por tanto de otra forma de socializar con el entorno más próximo, en ocasiones con gente conocida o incluso con personas a la que previamente ni se conocía.

Y con los vecinos, cuando sales a aplaudir. Te saludas de un balcón a otro, y estas un rato hablando y ya está. Ahora, incluso estando en la ventana los saludas. (U3) 
Es momento ahora de abordar específicamente el tema de las redes sociales. La tenencia y el uso del teléfono móvil son prácticamente indispensables en las sociedades actuales, y muy especialmente en los perfiles jóvenes que dedican un gran tiempo a su utilización. El teléfono móvil, Internet mediante, constituye la piedra angular sobre la que se articulan prácticamente todas sus relaciones sociales, tanto con amistades como con colegas de clase. Aunque no toda la población joven disfruta del mismo acceso a las Nuevas Tecnologías, ni poseen el mismo grado de conocimiento en cuanto a sus competencias para obtener un correcto aprovechamiento de las mismas (Calderón, 2020).

Todas las personas entrevistadas tienen presencia en al menos una red social, además de contar con alguna aplicación de mensajería instantánea; incluso los perfiles que se declaran abiertamente menos partidarios de las nuevas tecnologías o que afirman que no prestan mucha atención al teléfono móvil. Las redes sociales en las que se encuentran principalmente son: Facebook, Twitter o Instagram, con intencionalidad de uso diferenciada. Teniendo algunas personas cuenta en todas ellas, cuestión que evidencia que las redes sociales son esenciales en este perfil -independientemente de las posibles fluctuaciones por los rangos de edad-, ya que les permiten estar informados y ante todo conectados entre sí.

En la medida en que los medios de comunicación empleados son digitales, la presencia de las pantallas es omnipresente durante el confinamiento. Las pantallas durante este lapso de tiempo constituyen un elemento catalizador de diferentes áreas que son claramente esenciales para este grupo: educación, socialización, información, entretenimiento, etc. Esto se refleja de forma palpable en el número de horas que se invierte frente a dispositivos electrónicos, que es muy superior respecto al uso previo al confinamiento, ya que muchos ámbitos de la vida quedan supeditados a su utilización. Por lo tanto, ante la imposibilidad de realizar actividades en el exterior, la ventana que se abre al mundo tiene forma de pantalla. De ahí que el número de horas que se está frente a ordenadores, tabletas, móviles y en última instancia televisor sea muy notorio.

Yo muchas veces me tiro en frente del ordenador toda la mañana y toda la tarde. Y por la noche jugando con el móvil. $Y$ si hablas con los amigos tres horas es delante del ordenador. Diez horas. Los apuntes los llevamos en ordenador. Y si estás estudiando seis horas, son seis horas de ordenador. (U3)

Sin embargo, a pesar de las ventajas que lleva aparejado el disponer de estos recursos, su gran dependencia supone un problema en la medida en que se sobreexplota su utilización. Esto deriva en algunas problemáticas evidentes que han señalado muchas personas: pérdida de visión a corta distancia, dolores de cabeza frecuentes, dificultades para conciliar el sueño, etc. El papel actual de los nuevos medios de comunicación como agentes socializadores en nuestra sociedad es evidente y se ve reflejado en la cultura de pantalla. De ahí el notorio protagonismo de las herramientas tecnológicas, y especialmente de determinadas formas de comunicación, que están marcando los procesos de socialización de los más jóvenes de nuestra sociedad (Jiménez y Aroca, 2019) 
Yo creo que ha sido todo el día. A veces no, pero todos los ratos videollamadas, móvil, WhatsApp, Instagram, Teams... Siempre frente a una pantalla. Lo que conlleva que todo el mundo tenga dolor de cabeza, insomnio... Yo creo que ha tenido mucho que ver también porque ha cambiado el estilo de vida. (R7)

Hoy en día, se evidencia un auge del fenómeno denominado "pantallización”, según el cual, se están adaptando los medios de comunicación de masas, incluidos los tradicionales, a la pantalla para ser ofrecidos y consumidos. Además, la pantalla supone una parte fundamental de nuestras identidades, en la medida en que mediante su uso damos forma a nuestro yo y establecemos comunicaciones con los demás (Diez et al., 2020).

Yo creo que como es normal, al principio mucho más, (...) pero sí se ha visto ampliado el abanico de gente con la que he hablado y el tiempo. Incluso gente con la que llevaba tiempo sin hablar, he vuelto a hablar. Con amigos y amigas bastante y casi todas las semanas o casi todos los días. A través de WhatsApp, videollamadas un porrón, y con bastantes he tenido y sigo teniendo contacto. Ahora menos, pero sí. Y conversaciones con amigos los fines de semana, hacemos Zoom y echamos una cerveza. (U1)

El hecho de disponer este colectivo de bastante tiempo libre durante el confinamiento, potencia en cierto modo un mayor uso de los recursos tecnológicos. Aunque esta utilización llega a ser considerada como excesiva en algunos casos por diversos motivos.

Con amigos como estamos todo el rato con el móvil, creo que hablamos más por los grupos y que estamos más activos. WhatsApp y redes sociales. Instagram y Twitter. Al principio y antes de ponerme con el tema de estudios sí. Pero ahora como que me he cansado un poco del móvil. (R6)

Además, es importante resaltar la relevancia que se le da a las amistades en esta etapa vital, en cuanto a que pueden cumplir el rol de confidentes, algo que es especialmente valorado de forma positiva durante el confinamiento, una situación que lleva aparejado cierto desgaste emocional y psicológico.

Porque por muy bien que te lleves con tu familia, cuando necesitas contar algo, vas a tu hermana o a tus amigas o amigos. No es lo mismo hablar por WhatsApp (se refiere por escrito) que hacer una videollamada. Las demás personas también están agobiadas y nos alegra contarnos las cosas y vernos. (U2)

Una cuestión importante, es que este período de encierro ha permitido a algunas personas disponer de más tiempo para realizar actividades novedosas y sobre todo para efectuar un diálogo interno. Son muchas las personas que consideran que el confinamiento les ha brindado la posibilidad de disponer de un tiempo para estar con ellas mismas. Un tiempo que antes no hallaban por el ritmo de vida que llevaban. Este lapso temporal supone la posibilidad de recapacitar con una mayor perspectiva, siendo factible reflexionar sobre lo que sucede de una forma genérica pero también para conocer mejor su cercana realidad. Siendo este un aspecto muy positivo del 
confinamiento, que contribuye a la maduración personal e incluso a un mayor autoconocimiento.

Esto nos ha ayudado a ver de verdad quién queremos que forme parte de nuestra vida y quién no. Quién de verdad muestra interés, quién te importa y a quién debes llamar amigos. No nos dedicamos a veces tiempo a nosotros mismos porque se lo dedicamos a los demás. Y el confinamiento nos ha venido muy bien para parar un momento y decir: ¿Estoy haciendo las cosas bien? ¿Me gusto o no me gusto? Y analizarlo. (R1)

Sin embargo, un exceso de actividad reflexiva puede llevar aparejado un enfoque excesivamente crítico que puede llegar incluso a propiciar conflictos existenciales.

Creo que lo peor ha sido el estar pensando demasiado y no estar ocupada. No sé si me gusta mi carrera... Cosas que antes veías obvias o sí que siguen siendo obvias, pero que por la situación en la que estamos viviendo no es así. (U4)

A lo largo de este análisis se ha incidido en los usos del tiempo, así como lo que implica su gestión en relación con la actividad educativa. No obstante, en estas últimas líneas se propone un espacio para la reflexión personal a partir de ese análisis del tiempo existencial de cada uno durante la etapa del confinamiento, obteniendo cada uno/a sus propias deducciones.

Ahora tenemos todo el tiempo del mundo, pero no lo hemos aprovechado ni disfrutado porque realmente no lo tenías. A veces te das cuenta de las veces que has perdido el tiempo en cosas inútiles. (U4)

\section{| Conclusiones |}

Este trabajo constituye una modesta aportación al estudio de los usos del tiempo, con la peculiaridad de estar enmarcado en un contexto de gran excepcionalidad, auspiciado por la pandemia y focalizado en un confinamiento domiciliario sin precedentes.

A partir de los resultados alcanzados, se evidencia cómo la limitación de la movilidad espacial en este colectivo condiciona seriamente la organización de sus ritmos vitales, tanto en sus obligaciones como en lo referente a otros aspectos de sus vidas. Queda patente cómo se establece una ruptura abrupta en la forma de organizar las rutinas diarias, que viene marcada por la imposición del estado de alarma. En un momento inicial, predomina el desconcierto y la preocupación por las implicaciones de la crisis sanitaria. Se da mayor importancia a asimilar el cambio impuesto y a todo lo sucedido; así como a mantener activa la comunicación con familiares no convivientes y amistades o pareja. También destaca el atender a cuestiones de carácter puramente operativo, como puede ser la organización doméstica. 
Según la primera hipótesis planteada, el confinamiento estricto condiciona negativamente las formas de organización temporal del alumnado. En base a la información analizada, se rechaza esta primera hipótesis por los siguientes motivos que a continuación se detallan. El confinamiento por sí mismo no influye negativamente en las formas de organización temporales, aunque condiciona enormemente. El proceso adaptativo queda condicionado a tres factores: El hogar, la personalidad del sujeto y la continuidad de la docencia. En primer lugar, los medios y las características del hogar de que se dispone condicionan notablemente el poder llevar a cabo su labor de estudio con éxito. El tamaño y las características de la vivienda, así como el número de convivientes van a ser decisivos. En este sentido, no es lo mismo disponer de un espacio propio para el estudio que tener que compartirlo con otras personas. Ni tampoco tienen la misma calidad de vida durante el confinamiento las personas que disponen de un patio al que poder salir a tomar aire fresco y descansar que las que ni siquiera disponen de un balcón. En segundo lugar, destaca el grado de capacidad personal para gestionar el tiempo adecuadamente. Aquí, el factor de la autodisciplina es el que marca un mayor o menor éxito en la obtención de resultados. En tercer lugar, se encuentra un elemento organizador externo, un factor dinamizador como es la continuidad de la docencia mediante las clases en remoto. Este último agente, ayuda a todos los perfiles a retomar la actividad y los horarios previos, algo que contribuye a organizar los ritmos vitales y a dar un mayor sentido a la actividad estudiantil. La combinación de estos tres mecanismos posibilita en mayor o menor medida una adaptación más o menos exitosa al confinamiento.

La segunda hipótesis afirma que el confinamiento condiciona una percepción subjetiva de menor productividad. Se acepta esta hipótesis, pues, de forma generalizada, el alumnado no aprecia un avance significativo en su proceso de estudio, aunque se lleven a cabo grandes esfuerzos. Según queda evidenciado, el aislamiento domiciliario influye negativamente en la percepción de su eficacia respecto al estudio. Puesto que, por una parte, permanecer en el hogar dificulta la concentración por muy diferentes motivos; $y$ por otra parte, ante la imposibilidad de cambiar de entorno, no es posible desconectar y posteriormente retomar la actividad con nuevas energías.

Como propuestas de investigación, se plantean dos alternativas no excluyentes. Por una parte, estudiar los usos del tiempo de diferentes colectivos en base a grupos de edad para conocer mejor cómo se comporta nuestra sociedad y en que ámbitos centran esencialmente sus actividades. Por otra parte, en consonancia con el período actual de pandemia, se sugiere estudiar cómo viven diferentes colectivos el momento presente, desde una perspectiva de las emociones.

Para concluir, este análisis del enfoque temporal permite conocer de primera mano cómo vive el proceso educativo este alumnado universitario, cuestión que tiene utilidad para plantear alternativas educativas futuras en contextos de excepcionalidad. De este modo, se sugiere fomentar el aprendizaje con el alumnado, teniendo en cuenta sus circunstancias, a través de diferentes herramientas y recursos que les permitan optimizar sus tiempos. Cabe destacar aquí la importancia de la adaptación pedagógica y de los microaprendizajes así como de nuevas formas de evaluar (Zapata-Ros, 2018). Por otra 
parte, se pone de manifiesto la importancia de entender la educación de una forma consciente e interrelacionada con el mundo, compartiendo con el alumnado lo que está sucediendo y sus implicaciones (Candel, 2020).

\section{Referencias}

Área, M., y Pessoa, T. (2012). "De lo sólido a lo líquido: Las nuevas alfabetizaciones ante los cambios culturales de la Web 2.0". Comunicar, 38, 1320. https://doi.org/10.3916/C38-2011-02-01

Bayés, R. (2007). El reloj emocional. Barcelona: Alienta Editorial.

Cabero, J., y Valencia, R. (2021). Y el COVID-19 transformó al sistema educativo: reflexiones y experiencias por aprender. International Journal of Educational Research and Innovation (IJERI), 15, 218-228 ISSN: 2386-4303. https://doi.org/10.46661/ijeri.5246

Calderón, D. (2020). Jóvenes y desigualdad digital: las brechas de acceso, competencias y uso. Madrid: Centro Reina Sofía sobre Adolescencia y Juventud.

Candel, C. (2020). Hacia lo significativo. El diario de la educación. Recuperado de: https://eldiariodelaeducacion.com/2020/04/03/hacia-lo-significativo/

De Miguel, A., y De Miguel, I. (2014). La percepción de los españoles sobre el tiempo. Madrid: Centro de Investigaciones Sociológicas.

Díaz Herrera, C. (2018). Investigación cualitativa y análisis de contenido temático. Orientación intelectual de revista Universum. Revista General de Información y Documentación, 28 (1), 119-142.

Díez García, R., Belli, S., y Márquez, I. V. (2020). La COVID-19, pantallas y reflexividad social. Cómo el brote de un patógeno está afectando nuestra cotidianidad. Revista Española de Sociología, 29 (3), 759-768. http://dx.doi.org/10.22325/fes/res.2020.49

Díez-Gutiérrez, E., y Gajardo-Espinoza, K. (2020). Educar y evaluar en tiempos de coronavirus: la situación en España". Multidisciplinary Journal of Educational Research, 10(2), 102-134. https://doi.org/10.4471/remie.2020.5604

Feito, R. (2020). Este es el fin de la escuela tal y como la conocemos. Unas reflexiones en tiempo de confinamiento. Revista de Sociología de la Educación-RASE, 13(2) Especial, COVID-19, 156-163. http://dx.doi.org/10.7203/RASE.13.2.17130

Jiménez, G., y Aroca, F. (2019). "Masculinidades y feminidades en preadolescentes residentes en contextos rurales de Castilla-La Mancha". Revista de Sociología de la Educación-RASE, 12(1), 40-62. http://dx.doi.org/10.7203/RASE.12.1.13253 
Fernández, M. (2020). Sociología y Ciencias Sociales en tiempos de crisis pandémica. Revista de Sociología de la Educación-RASE, 13 (2) Especial, COVID-19, 105-113. http://dx.doi.org/10.7203/RASE.13.2.17113

García-Planas, M. I., y Taberna Torres, J. (2021). Transición de la docencia presencial a la no presencial en la UPC durante la pandemia del COVID-19. International Journal of Educational Research and Innovation (IJERI), 15, 177-187. https://doi.org/10.46661/ijeri.5015

Garzón, A., y Gil, J. (2018). Gestión del tiempo en alumnado universitario con diferentes niveles de rendimiento académico. Educação e Pesquisa, 44. https://doi.org/10.1590/s1678-4634201708157900

Pérez-López, E., Vázquez, A., y Cambero, S. (2021). Educación a distancia en tiempos de COVID-19: Análisis desde la perspectiva de los estudiantes universitarios. Revista Iberoamericana de Educación a Distancia, 24(1), 331-350. http://dx.doi.org/10.5944/ried.24.1.27855

Tarabini, A. (2020). ¿Para qué sirve la escuela? Reflexiones sociológicas en tiempos de pandemia global. Revista de Sociología de la Educación-RASE, 13 (2) Especial, COVID-19, 145-155. https://doi.org/10.7203/RASE.13.2.17135

Valles, M. (2000). Técnicas cualitativas de investigación social. Madrid: Síntesis.

Zapata-Ros, M. (2018). La universidad inteligente. La transición de los LMS a los Sistemas Inteligentes de Aprendizaje en Educación Superior. RED. Revista de Educación a Distancia, 57(10). https://www.um.es/ead/red/57/zapata2.pdf

\section{Anexos}

Tabla 1. Características de la persona entrevistada y de su hogar durante el confinamiento

\begin{tabular}{|c|c|c|c|c|c|c|c|c|c|}
\hline Orden de & & & $N^{\circ}$ de personas & Trabajadores/as & Perciben & Relación & Metros & Dispone & \\
\hline Entrevista & Edad & Género & en el hogar & en activo & prestación & convivientes & cuadrados & de patio & Código \\
\hline 1 & 40 & Hombre & 1 & 1 & No & - & 70 & No & UI \\
\hline 2 & 21 & Mujer & 3 & 0 & $\mathrm{Si}$ & Parentesco & 90 & $\mathrm{Si}$ & RI \\
\hline 3 & 18 & Mujer & 4 & 0 & $\mathrm{Si}$ & Parentesco & 90 & $\mathrm{Si}$ & R2 \\
\hline 4 & 20 & Mujer & 4 & 0 & $\mathrm{Si}$ & Parentesco & 90 & No & U2 \\
\hline 5 & 18 & Mujer & 4 & 1 & $\mathrm{Si}$ & Parentesco & 80 & No & U3 \\
\hline 6 & 21 & Mujer & 2 & 0 & No & Amistad & 100 & No & U4 \\
\hline 7 & 28 & Hombre & 4 & 1 & $\mathrm{Si}$ & Parentesco & 75 & No & U5 \\
\hline 8 & 22 & Mujer & 4 & 2 & No & Parentesco & 120 & $\mathrm{Si}$ & R3 \\
\hline 9 & 19 & Mujer & 4 & 1 & $\mathrm{Si}$ & Parentesco & 70 & No & U6 \\
\hline 10 & 18 & Hombre & 4 & 2 & No & Parentesco & 90 & $\mathrm{Si}$ & R4 \\
\hline 11 & 21 & Hombre & 4 & 1 & No & Amistad & 120 & No & U7 \\
\hline 12 & 20 & Mujer & 2 & 1 & No & Parentesco & 120 & $\mathrm{Si}$ & บ8 \\
\hline 13 & 22 & Hombre & 4 & I & $\mathrm{Si}$ & Pareja y sus padres & 120 & $\mathrm{Si}$ & U9 \\
\hline 14 & 21 & Mujer & 4 & 2 & No & Parentesco & 100 & $\mathrm{Si}$ & R5 \\
\hline
\end{tabular}

Fuente: elaboración propia 


\section{| Nota biográfica |}

Francisco Javier Aroca Cifuentes es licenciado en Sociología por la Universidad de Alicante (España), posteriormente realiza el Máster en Problemas Sociales del Departamento de Sociología III de la Facultad de Ciencias Políticas y Sociología de la Universidad Nacional de Educación a Distancia (UNED). Actualmente es doctorando en la Universidad de Castilla-La Mancha (España), siendo el tema de su tesis: las nuevas formas de pobreza y exclusión social.

Su experiencia profesional se ha centrado principalmente en el ámbito de la investigación social, trabajando para diferentes entidades públicas y privadas. Desde 2003 ha participado en numerosos estudios sociales y de mercado, coordinando diversas investigaciones en el ámbito territorial de Castilla-La Mancha. Sus principales líneas de investigación se centran en: inmigración, género, exclusión social y juventud. Además, en los últimos años ha presentado comunicaciones en diferentes Jornadas y Seminarios, tanto nacionales como internacionales, enmarcadas en las siguientes temáticas: género, juventud, exclusión social, deporte y Covid-19.

Actualmente imparte docencia en la Universidad de Castilla-La Mancha, concretamente en la Facultad de Ciencias de la Educación y Humanidades de Cuenca y en la Facultad de Derecho de Albacete. Asimismo, es profesor tutor de asignaturas de Sociología en el Centro Asociado de Cartagena (UNED).

\section{| Agradecimientos |}

Aprovecho estas líneas para trasladar un agradecimiento muy especial a los y las protagonistas de las narrativas analizadas: todas las personas que han colaborado desinteresadamente con su tiempo, experiencias personales e ideas en esta investigación. Sin su participación no hubiese sido posible llevar a cabo este trabajo. Gracias, también, por permitirme el establecimiento de un espacio de diálogo cercano y sin trabas, que en definitiva ha servido para alcanzar una gran proximidad en un momento extraño y a pesar de la distancia existente de por medio. 